\title{
RESONANCE FREE REGIONS AND NON-HERMITIAN SPECTRAL OPTIMIZATION FOR SCHRÖDINGER POINT INTERACTIONS
}

\author{
Sergio Albeverio And Illya M. Karabash
}

\begin{abstract}
Resonances of Schrödinger Hamiltonians with point interactions are considered. The main object under the study is the resonance free region under the assumption that the centers, where the point interactions are located, are known and the associated "strength" parameters are unknown and allowed to bear additional dissipative effects. To this end we consider the boundary of the resonance free region as a Pareto optimal frontier and study the corresponding optimization problem for resonances. It is shown that upper logarithmic bound on resonances can be made uniform with respect to the strength parameters. The necessary conditions on optimality are obtained in terms of first principal minors of the characteristic determinant. We demonstrate the applicability of these optimality conditions on the case of 4 equidistant centers by computing explicitly the resonances of minimal decay for all frequencies. This example shows that a resonance of minimal decay is not necessarily simple, and in some cases it is generated by an infinite family of feasible resonators.
\end{abstract}

Mathematics subject classification (2010): 35J10, 35B34, 35P15, 49R05, 90C29, 47B44.

Keywords and phrases: Exponential polynomial, Pareto optimal design, high-Q cavity, quasi-normal eigenvalue, scattering pole, delta-interaction, zero-range.

\section{REFERENCES}

[1] S. Albeverio, J. E. Fenstad, R. Høegh-Krohn, Singular perturbations and nonstandard analysis, Trans. Amer. Math. Soc. 252 (1979), 275-295.

[2] S. Albeverio, F. GeszTesY, R. HøEGH-KROHN, The low energy expansion in nonrelativistic scattering theory, in Annales de l'IHP Physique théorique, vol. 37, no. 1, 1982, 1-28.

[3] S. Albeverio, F. Gesztesy, R. Høegh-Krohn, H. Holden, Solvable models in quantum mechanics, 2nd edition, with an appendix by P. Exner, AMS Chelsea Publishing, Providence, RI, 2005.

[4] S. Albeverio, F. Gesztesy, R. HøeGh-Krohn, L. Streit, Charged particles with short range interactions, in Annales de l'IHP Physique théorique, vol. 38, no. 3, 1983, 263-293.

[5] S. Albeverio, R. Høegh-Krohn, Perturbation of resonances in quantum mechanics, J. Math. Anal. Appl. 101 (1984), 491-513.

[6] S. AlbeVerio, P. KuRAsov, Singular perturbations of differential operators: solvable Schrödingertype operators, Cambridge University Press, 2000.

[7] C. A. Berenstein, R. Gay, Complex analysis and special topics in harmonic analysis, Springer Science \& Business Media, 2012.

[8] S. Boyd, L. Vandenberghe, Convex optimization, Cambridge university press, Cambridge, 2004.

[9] S. J. Cox, M. L. Overton, Perturbing the critically damped wave equation, SIAM J. Appl. Math. 56 (1996), 1353-1362.

[10] S. CoX, E. ZuAZUA, The rate at which energy decays in a string damped at one end, Indiana Univ. Math. J. 44 (1995), 545-573.

[11] E. B. DAVIES, P. EXNER, J. LipovskÝ, Non-Weyl asymptotics for quantum graphs with general coupling conditions, Journal of Physics A 43 (47) (2010), 474013.

[12] U. P. Dharanipathy, M. Minkov, M. Tonin, V. Savona, R. Houdré, High-Q silicon photonic crystal cavity for enhanced optical nonlinearities, Applied Physics Letters 105 (2014), 101101. 
[13] S. Dyatlov, M. Zworski, Mathematical theory of scattering resonances, Book in progress, http://math. mit. edu/dyatlov/res/.

[14] P. EXNER, An isoperimetric problem for point interactions, J. Phys. A 38 (2005), 4795.

[15] P. EXNER, Open quantum systems and Feynman integrals, Springer Science \& Business Media, Berlin, 2012.

[16] P. ExNER, M. FraAs, E. M. HARRELl, On the critical exponent in an isoperimetric inequality for chords, Physics Letters A 368 (2007), 1-6.

[17] P. EXNER, E. M. HARRELl, M. Loss, Inequalities for means of chords, with application to isoperimetric problems, Lett. Math. Phys. 75 (2006), 225-233.

[18] P. Exner, V. Lotoreichik, A spectral isoperimetric inequality for cones, Lett. Math. Phys. 107 (2017), 717-732.

[19] R. Froese, Asymptotic distribution of resonances in one dimension, J. Differential Equations 137 (1997), 251-272.

[20] F. GESZTES Y, Perturbation theory for resonances in terms of Fredholm determinants, in ResonancesModels and Phenomena, edited by S. Albeverio, L. S. Ferreira and L. Streit, Springer, BerlinHeidelberg, 1984, 78-104.

[21] Yiqi Gu, Xiaoliang Cheng, A Numerical Approach for Defect Modes Localization in an Inhomogeneous Medium, SIAM J. Appl. Math. 73 (2013), 2188-2202.

[22] E. M. Harrell, General lower bounds for resonances in one dimension, Comm. Math. Phys. 86 (1982), 221-225.

[23] E. M. Harrell, R. Svirsky, Potentials producing maximally sharp resonances, Trans. Amer. Math. Soc. 293 (1986), 723-736.

[24] P. Heider, D. Berebichez, R. V. Kohn, and M. I. Weinstein, Optimization of scattering resonances, Struct. Multidisc. Optim. 36 (2008), 443-456.

[25] C.-Y. KAO, F. SANTOSA, Maximization of the quality factor of an optical resonator, Wave Motion 45 (2008), 412-427.

[26] I. M. KARABASH, Optimization of quasi-normal eigenvalues for 1-D wave equations in inhomogeneous media; description of optimal structures, Asymptotic Analysis 81 (2013), 273-295.

[27] I. M. KARABASH, Pareto optimal structures producing resonances of minimal decay under $L^{1}$-type constraints, J. Differential Equations 257 (2014), 374-414.

[28] I. M. Karabash, O. M. Logachova, I. V. Verbytskyi, Nonlinear bang-bang eigenproblems and optimization of resonances in layered cavities, Integr. Equ. Oper. Theory 88 (1) (2017), 15-44.

[29] I. M. KARABASH, O. M. LogACHOVA, I. V. VERBYTSKYi, Overdamped modes and optimization of resonances in layered cavities, to appear in Methods of Functional Analysis and Topology.

[30] T. KATO, Perturbation theory for linear operators, Springer Science \& Business Media, Berlin, 2013.

[31] V. Kostrykin, R. Schrader, Kirchhoff's rule for quantum wires, Journal of Physics A 32 (4), (1999), 595.

[32] T. Kottos, U. Smilansky, Quantum graphs: a simple model for chaotic scattering, Journal of Physics A 36 (12), (2003), 3501-3524.

[33] P. D. LAX, R. S. PhILliPS, A logarithmic bound on the location of the poles of the scattering matrix, Arch. Ration. Mech. Anal. 40 (1971), 268-280.

[34] X. Liang, S. G. Johnson, Formulation for scalable optimization of microcavities via the frequencyaveraged local density of states, Optics express 21 (2013), 30812-30841.

[35] R. E. LANGER, On the zeros of exponential sums and integrals, Bulletin of the American Mathematical Society 37 (4), (1931), 213-239.

[36] V. LotoREICHIK, Spectral isoperimetric inequalities for $\delta$-interactions on open arcs and for the Robin Laplacian on planes with slits, (2016), arXiv preprint arXiv:1609.07598.

[37] B. Maes, J. Petráček, S. Burger, P. Kwiecien, J. Luksch, I. Richter, Simulations of highQ optical nanocavities with a gradual 1D bandgap, Optics express 21 (2013), 6794-6806.

[38] G. Mora, J. M. Sepulcre, T. Vidal, On the existence of exponential polynomials with prefixed gaps, Bulletin of the London Mathematical Society 45 (6), (2013), 1148-1162.

[39] M. Notomi, E. Kuramochi, H. TAniyama, Ultrahigh-Q nanocavity with 1D photonic gap, Optics Express 16 (15) (2008), 11095-11102.

[40] KAI OGASAWARA, The analysis of resonant states in a one-dimensional potential of quantum dots, Honours Physics Bachelor thesis, University of British Columbia, 2014.

[41] B. Osting, M. I. WeInstein, Long-lived scattering resonances and Bragg structures, SIAM J. 
Appl. Math. 73 (2013), 827-852.

[42] V. PivOVARCHIK, H. WORACEK, Eigenvalue asymptotics for a star-graph damped vibrations problem, Asymptotic Analysis 73 (3), (2011), 169-185.

[43] M. Reed, B. Simon, Analysis of Operators, vol. IV of Methods of Modern Mathematical Physics, Academic Press, New York, 1978.

[44] A. A. Shushrov, Structure of resonances for symmetric scatterers, Theoretical and Mathematical Physics 64 (1985), 944-949.

[45] R. SVIRSKY, Maximally resonant potentials subject to p-norm constraints, Pacific J. Math. 129 (1987), 357-374.

[46] B. R. VAInBERG, Eigenfunctions of an operator that correspond to the poles of the analytic continuation of the resolvent across the continuous spectrum, (Russian) Mat. Sb. (N. S.) 87 (129) (1972), 293-308, English transl.: in Mathematics of the USSR-Sbornik, 16 (2) (1972).

[47] M. ZWorS KI, Sharp polynomial bounds on the number of scattering poles, Duke Math. J. 59 (1989), 311-323. 